\title{
Novel synergic antidiabetic effects of Astragalus polysaccharides combined with Crataegus flavonoids via improvement of islet function and liver metabolism
}

\author{
KAI CUI ${ }^{1,2^{*}}$, SHAOBO ZHANG ${ }^{3 *}$, XIN JIANG ${ }^{1,2}$ and WEIDONG XIE ${ }^{1,2}$ \\ ${ }^{1}$ School of Life Sciences, Tsinghua University, Beijing 100084; ${ }^{2}$ Shenzhen Key Lab of Health Science and Technology, \\ Division of Life Science and Health, Graduate School at Shenzhen, Tsinghua University, Shenzhen, Guangdong 518055; \\ ${ }^{3}$ Department of Clinical Laboratory, The First Affiliated Hospital of Shantou University Medical College, \\ Shantou, Guangdong 515041, P.R. China
}

Received May 24, 2015; Accepted February 22, 2016

DOI: $10.3892 / \mathrm{mmr} .2016 .5140$

\begin{abstract}
The present study investigated the synergic effects and potential mechanisms of action of Astragalus polysaccharides (APS) combined with Crataegus flavonoids (CF) in the treatment of type 1 diabetes. Diabetes was induced by intraperitoneal injection of $100 \mathrm{mg} / \mathrm{kg}$ streptozotocin in mice. Normal and untreated diabetic control mice were used, and CF-treated (200 mg/kg/day), APS-treated (200 mg/kg/day), APS + CF (AC)-treated (200 mg/kg/day of each) and metformin-treated ( $200 \mathrm{mg} / \mathrm{kg} / \mathrm{day}$ ) diabetic mice were orally administrated the appropriate therapeutic agent for 4 weeks. The results demonstrated that AC treatment significantly reduced the fasting blood glucose, food and water intake in the diabetic mice. The AC group demonstrated increased serum insulin levels and islet cell function was restored. Furthermore, the AC-treated mice demonstrated significant increases in the protein expression levels of pancreatic and duodenal homeobox-1 and phosphorylated adenosine 5'-monophosphate-activated protein kinase in
\end{abstract}

Correspondence to: Dr Weidong Xie, Shenzhen Key Lab of Health Science and Technology, Division of Life Science and Health, Graduate School at Shenzhen, Tsinghua University, 2279 Lishui Road, Shenzhen, Guangdong 518055, P.R. China

E-mail: xiewd@sz.tsinghua.edu.cn

${ }^{*}$ Contributed equally

Abbreviations: AC, Astragalus polysaccharides combined with Crataegus flavonoids; AMPK, adenosine 5'-monophosphate-activated protein kinase; APS, Astragalus polysaccharides; AUC, area under the curve; CCL2, chemokine (C-C motif) ligand 2; CF, Crataegus flavonoids; IL-6, interleukin 6; PDX-1, pancreatic and duodenal homeobox-1; pAMPK, phosphorylated AMPK; OGTT, oral glucose tolerance test; STZ, streptozotocin; TCM, traditional Chinese medicine; TNF- $\alpha$, tumor necrosis factor- $\alpha$

Key words: diabetes, inflammation, fructus Crataegi pinnatifidae, Astragalus membranaceus (Fisch.) Bunge, pancreatic and duodenal homeobox-1, adenosine 5'-monophosphate-activated protein kinase the pancreatic and liver tissue samples, respectively. In addition, AC significantly increased the mRNA expression levels of neurogenin 3, v-maf musculoaponeurotic fibrosarcoma oncogene family, protein A and insulin, and simultaneously decreased the expressions of interleukin 6, tumor necrosis factor- $\alpha$ and chemokine (C-C motif) ligand 2 in the pancreatic islet cells of diabetic mice. The anti-inflammatory activity of APS and the islet-restoring effect of CF may contribute to the improvement of islet function. AC exerted greater antidiabetic effects compared with APS or CF treatments alone. These results indicated that $\mathrm{AC}$ treatment had a synergic antidiabetic effect, which may involve improvements in islet function and liver metabolism. These effects of AC may facilitate the treatment of type 1 or 2 diabetes, as these patients frequently experience impaired islet function and disordered extrapancreatic metabolism.

\section{Introduction}

Diabetes mellitus is a chronic disease characterized by the impairment of islet $\beta$-cell function, and dysfunctional glucose and lipid metabolism. Approximately $10 \%$ of the adults in China suffer from diabetes (1) and the incidence rate is rapidly increasing. Patients with diabetes mellitus suffer from macrovascular and microvascular complications (2). The currently available antidiabetic therapeutic agents exert significant hypoglycemic effects, however, many of these therapeutic agents require further development for an improved therapeutic outcome (3). The majority of hypoglycemic therapeutic agents do not rehabilitate islet $\beta$-cell function (4). For example, metformin (MF) is one of the therapeutic agents prescribed first to improve glucose metabolism; however, few studies have demonstrated improvements in islet cell function (5). Dipeptidyl peptidase-4 inhibitors and glucagon-like peptide-1 receptor agonists appear to have potential effects on the restoration of islet cells, however, are occasionally associated with severe side effects (6). Therapeutic agents that improve extrapancreatic glucose metabolism and rehabilitate islet cell function may provide a cure for type 1 or 2 diabetes. 
Traditional Chinese medicines (TCMs) are a significant alternative treatment modality, which may be administered to prevent and treat diabetes (7). These medicinal plants have comparable effects to common antidiabetic therapeutic agents and are consumed at a lower cost. Astragalus membranaceus has been documented in China as a tonic herb in TCM for thousands of years (8), and $\sim 80 \%$ of the TCM formulas supplied by the State Food and Drug Administration in China describe this plant as an antidiabetic therapeutic agent (7). Fructus Crataegi Pinnatifidae (hawthorn) is traditionally used to aid digestion and this plant is prescribed in 50\% of TCM formulas for the treatment of hyperlipidemia (9). In addition, hawthorn administration has antidiabetic effects (10). However, Astragalus or hawthorn were frequently used, as crude or low-quality extracts, in combination with other herbs to treat diabetes, which limited its further application in patients with diabetes as a result of poor quality controls and variable effects.

Combining two or more active components from TCMs, rather than using crude or low-quality herb extracts, to create a novel formula has become a popular method for developing therapeutic agents to treat diseases (11). Astragalus polysaccharides (APS) and Crataegus flavonoids (CF) are typical active components of Astragalus membranaceus and Fructus Crataegi Pinnatifidae, respectively. Furthermore, polysaccharides and flavonoids are the two primary natural components that exert potential antidiabetic effects. Combining the different natural components may reduce the respective adverse effects and enhance the common antidiabetic effects.

In China, Astragalus membranaceus and Fructus Crataegi Pinnatifidae are the most frequently administered herbs to treat diabetes and hyperlipidemia, respectively; therefore the present study hypothesized that APS combined with CF may have a synergic antidiabetic effect. The current study demonstrated that APS + CF (AC), rather than the combination of crude or low-quality extracts, yielded a significant, synergic antidiabetic effect by restoring $\beta$-islet cell function and enhancing the metabolism of liver tissues in streptozotocin (STZ)-induced type 1 diabetic mice.

\section{Materials and methods}

Animals. Male NIH Swiss outbred mice ( $\mathrm{n}=80,18 \pm 2 \mathrm{~g}, 4$ weeks old) were purchased from the Guangdong Medical Laboratory Animal Center (Foshan, China). The animals were kept in an environmentally controlled breeding room (temperature, $20 \pm 2^{\circ} \mathrm{C}$; humidity, $60 \pm 5 \%$; 12 -h light/dark cycle). The mice were fed with standard laboratory chow and water ad libitum. The study was performed in strict accordance with the recommendations of the Guide for the Care and Use of Laboratory Animals by the Institutional Animal Care and Use Committee of Tsinghua University (Beijing, China). The protocol was approved by the Animal Welfare and Ethics Committee of Tsinghua University. All animals were fasted from 09:00 to 15:00 prior to starting the trials.

Therapeutic agents. The flavonoids $(30 \%, \mathrm{~g} / \mathrm{g})$ from the $\mathrm{CF}$ fruits and polysaccharides $(50 \%, \mathrm{~g} / \mathrm{g})$ from the APS roots were purchased from Nanjing Zelang Medical Technology Co., Ltd. (Nanjing, China). The therapeutic agents were weighed for trials according to the net weights of flavonoids and polysaccharides. APS and CF were combined at the net weight ratio of $1 \mathrm{~g}$ polysaccharide to $1 \mathrm{~g}$ flavonoids, forming a novel formula designated as AC: $1 \mathrm{~g} \div 30 \%=3.33 \mathrm{~g}$ of CF extracts for net CF flavonoids; $1 \mathrm{~g} \div 50 \%=2 \mathrm{~g}$ of APS extracts for net APS polysaccharides. In addition, MF was purchased from Sigma-Aldrich (St. Louis, MO, USA). The therapeutic agents were freshly suspended in distilled water prior to administration.

Experimental procedures. Male NIH Swiss outbred mice were fasted for $24 \mathrm{~h}$ (weight, $18 \pm 2 \mathrm{~g}$ following fasting) and diabetes was induced by intraperitoneal (i.p.) injection of $100 \mathrm{mg} / \mathrm{kg}$ STZ (Sigma-Aldrich). STZ was freshly prepared in $0.1 \mathrm{M}$ of ice-cold citric acid buffer ( $\mathrm{pH}$ 4.5; Tianjin Baishi Chemical Industry Co., Ltd., Tianjin, China). One week after the STZ injection, the diabetic mice models with fasting blood glucose $>11.1 \mathrm{mmol} / 1$ were selected for further trials. The selected mice were divided into six groups $(\mathrm{n}=10)$ as follows: Normal and diabetic controls, and diabetic mice treated with $200 \mathrm{mg} / \mathrm{kg} / \mathrm{day}$ of APS, CF, AC and MF. Normal mice received no treatment and were fed with the identical volume of vehicles as diabetic controls. The therapeutic agents were freshly prepared and orally administrated twice a day at $0.3 \mathrm{ml} /$ mouse. Normal and diabetic control mice were treated with the identical volume of distilled water. Food and water intake were periodically measured in metabolic cages within $24 \mathrm{~h}$ and blood glucose was assayed once a week. Subsequent to 4 weeks of therapeutic agent administration, mice were fasted for $6 \mathrm{~h}$, anesthetized by i.p. injection with $10 \%(\mathrm{~g} / \mathrm{ml})$ urethane (Sigma-Aldrich) in phosphate-buffered saline (PBS) at a dosage of $0.1 \mathrm{ml} / 10 \mathrm{~g}$ body weight and blood samples $(\sim 0.5 \mathrm{ml})$ were collected for further biochemical assays. Subsequent to the mice sacrifice by cervical dislocation, liver and pancreatic tissues were removed and stored at $-80^{\circ} \mathrm{C}$, or soaked in $10 \%$ formalin solution (Tianjin Baishi Chemical Industry Co., Ltd.) for further biochemical and histopathological assays.

Oral glucose tolerance test (OGTT). The OGTT was conducted as previously described with slight modifications (12). Following therapeutic agent administration for 4 weeks, the mice $(n=6)$ were fasted for $6 \mathrm{~h}$. Blood samples $(20-50 \mathrm{ul})$ were collected from the tail veins to determine the blood glucose levels at $0,0.5,1$ and $2 \mathrm{~h}$ following $2.5 \mathrm{~g} / \mathrm{kg}$ glucose (Yongda Chemical Reagent Development Center, Tianjin, China) administration. The blood glucose concentration underwent temporal analysis, and the area under the curve (AUC) of blood glucose and time was calculated as follows: $A C_{0-2 h}=\left[\left(G_{0 h}+G_{0.5 h}\right) \times 0.5+\left(G_{0.5 h}+G_{1 h}\right) \times 0.5+\left(G_{1 h}+G_{2 h}\right) \times 1\right] / 2$, where $\mathrm{G}$ is the blood glucose value.

Hematoxylin and eosin $(H \& E)$ staining. Five mice were randomly selected from each group. The pancreatic tissue mass ( 2-mm diameter) of each mouse was fixed with $10 \%$ formaldehyde (v/v; Tianjin Baishi Chemical Industry Co., Ltd.) at room temperature for 2 weeks, and processed for routine paraffin-wax histology and staining with $\mathrm{H} \& \mathrm{E}$ by Dr Yunan Zhao at the Nanjing University of Traditional Chinese Medicine (Nanjing, China). Five continuous slices (10 $\mu \mathrm{m}$ thickness; distance between each slice, $20 \mu \mathrm{m}$ ) were 
obtained to measure the morphology of Langerhans islets in the pancreatic tissue of mice using the ECLIPSE TE2000-E inverted microscope (Nikon Corporation, Tokyo, Japan).

Western blotting. Pancreatic and liver tissues from five randomly selected mice from each group were homogenized and lysed with a mixture of $100 \mathrm{mM} \mathrm{NaCl}, 20 \mathrm{mM}$ Tris- $\mathrm{HCl}, 0.5 \mathrm{mM}$ EDTA (Sangon Biotech Co., Ltd., Shanghai, China) and $0.5 \%$ (v/v) Nonidet P-40 (Santa Cruz Biotechnology, Inc., Dallas, TX, USA). The lysates were centrifuged at $10,000 \times \mathrm{g}$ at $4^{\circ} \mathrm{C}$ for $10 \mathrm{~min}$. Supernatants were collected and the protein concentration was determined using the bicinchoninic acid assay kit (Nanjing Jiancheng Biotech Institute Co., Ltd., Nanjing, China). Western blot analysis was performed according to the manufacturer's protocol. Antibodies against rabbit polyclonal pancreatic and duodenal homeobox-1 (PDX-1; 1:500 to 1:1,000; Abcam, Cambridge, UK; cat. no. ab47267), rabbit monoclonal adenosine 5'-monophosphate (AMP)-activated protein kinase (AMPK) and phosphorylated AMPK (pAMPK; 1:500 to 1:1,000; Cell Signaling Technology, Inc., Danvers, MA, USA; cat. nos. 5831 and 4188, respectively), and mouse monoclonal $\beta$-actin (1:500 to 1:1,000; Santa Cruz Biotechnology, Inc.; cat. no. sc-47778) were used for overnight incubation at $4{ }^{\circ} \mathrm{C}$ for protein targeting. Protein expression was visualized with horseradish peroxidase-conjugated goat anti-rabbit $(1: 2,000$; Amersham Biosciences; GE Healthcare Bio-Sciences, Pittsburgh, PA, USA; cat. no. RPN4301) and goat anti-mouse (1:5,000; Abcam; cat. no. ab97040) secondary antibodies, and the enhanced LumiGLO chemiluminescent substrate kit (KPL, Inc., Gaithersburg, MD, USA). The protein expression of $\beta$-actin was used as the reference expression for normalization and data were analyzed using the ImageJ software, version 1.48 (National Institutes of Health, Bethesda, MD, USA).

Reverse transcription-quantitative polymerase chain reaction $(R T-q P C R)$. Five mice were randomly selected from each group. Total RNAs of pancreatic islet tissues $(\sim 50 \mathrm{mg})$ were extracted using RNAiso Plus reagent (Takara Biotechnology Co., Ltd., Dalian, China) according to the manufacturer's instructions. RT was performed using the PrimeScript Reagent kit (Takara; cat. no. DRR037A) according to the manufacturer's instructions. The RT reaction solution $(10 \mu \mathrm{l}$ total) was consisted of $2 \mu 15 \mathrm{X}$ PrimeScript Buffer, $0.5 \mu \mathrm{l}$ PrimeScript RT Enzyme Mix I, $0.5 \mu$ l Oligo dT Primer (50 $\mu \mathrm{M}), 0.5 \mu \mathrm{l}$ Random 6 mers $(100 \mu \mathrm{M}), 1 \mu \mathrm{g}$ total RNA and RNase free water. The procedure was performed with the Alpha Unit Block Assembly for DNA Engine systems (Bio-Rad Laboratories, Inc., Hercules, CA, USA) with a thermocycler program comprising steps at $42^{\circ} \mathrm{C}$ for $30 \mathrm{~min}$, $85^{\circ} \mathrm{C}$ for $5 \mathrm{~min}$, and a hold step at $4^{\circ} \mathrm{C}$. The genes were selected for RT-qPCR and the primers were synthesized by Invitrogen (Thermo Fisher Scientific, Inc., Waltham, MA, USA; Table I). $\beta$-actin served as the internal control for normalization. qPCR was conducted using the SYBR Green I real-time PCR Master Mix according to the manufacturer's protocol (Toyobo Co, Ltd., Osaka, Japan; cat. no. QPT-201) and using the Applied Biosystems 7300 real-time PCR System (Thermo Fisher Scientific, Inc.). The qPCR reaction solution (20 $\mu \mathrm{l}$ total) was consisted of $6.4 \mu \mathrm{l}$ distilled water, $10 \mu \mathrm{l} \mathrm{SYBR}$ Green Realtime PCR Master Mix, $0.8 \mu \mathrm{l}$ forward primer $(10 \mu \mathrm{M}), 0.8 \mu 1$ reverse primer $(10 \mu \mathrm{M})$ and $2 \mu \mathrm{l}$ cDNA. The procedure involved pre-denaturation of cDNA samples at $95^{\circ} \mathrm{C}$ for $60 \mathrm{sec}$ (first stage) and the amplification of the denatured cDNA samples with 40 cycles at $95^{\circ} \mathrm{C}$ for $15 \mathrm{sec}$, at $60^{\circ} \mathrm{C}$ for $15 \mathrm{sec}$ and at $72^{\circ} \mathrm{C}$ for $45 \mathrm{sec}$ (second stage). Data were normalized using the $\beta$-actin gene and fold changes were calculated using the $2^{-\Delta \Delta \mathrm{Cq}}$ normalization method (13).

Statistical analysis. Data are expressed as the mean \pm standard deviation. The comparison of the mean values for the groups was performed using one-way analysis of variance. Newman-Keuls comparisons were used to determine the source of significant differences and Pearson correlation analysis was conducted using SPSS software (version 12.0; SPSS, Inc., Chicago, IL, USA) when appropriate. $\mathrm{P}<0.05$ was considered to indicate a statistically significant difference.

\section{Results}

Body weight, and food and water intake. The diabetic syndromes in the STZ-induced mice were investigated. The body weights of diabetic mice were significantly reduced at 0 $(\mathrm{P}<0.01), 17$ and 24 days $(\mathrm{P}<0.05)$ compared with the normal control (Fig. 1A). AC-treated mice exhibited significantly increased body weights at 10 days, and APS- and CF-treated mice demonstrated significantly reduced body weights at 21 and 24 days, and 24 and 28 days, respectively, compared with the diabetic controls (Fig. $1 \mathrm{~A} ; \mathrm{P}<0.05)$. AC and $\mathrm{CF}$ treatment was not able to effectively reverse the decrease of body weights in this diabetic model, as AC and CF likely exert a similar body-lowering effect as MF treatment.

Furthermore, the diabetic mice demonstrated an increase in food intake after 7 days when compared with the normal controls (Fig. 1B). In addition, the AC-, CF- and MF-treated mice attenuated the increase in food intake following 7 days of treatment (Fig. 1B). The APS-treated mice did not demonstrate a significant change in food intake compared with the diabetic control (Fig. 1B; P>0.05). When comparing the normal control and diabetic mice a significant increase in water intake was demonstrated during the period 4 weeks of observation (Fig. 1C). The AC- and CF-treated mice significantly attenuated the increase in the water intake of diabetic mice following 10 days of treatment compared with the diabetic controls (Fig. 1C; $\mathrm{P}<0.01$ and $\mathrm{P}<0.05$, respectively). The MF- and APS-treated mice did not demonstrate significant changes in the water intake.

Fasting blood glucose and serum insulin. The diabetic mice demonstrated a significant increase of fasting blood glucose compared with the normal control (Fig. 1D; $\mathrm{P}<0.05$ ). AC- and MF-treated mice demonstrated a significant decrease in fasting blood glucose compared with the diabetic controls at 3 and 4 weeks of treatment (Fig. 1D; $\mathrm{P}<0.05$ and $\mathrm{P}<0.01$, respectively). The APS-treated mice demonstrated a significant increase compared with the diabetic controls at 3 and 4 weeks of treatment (Fig. 1D; $\mathrm{P}<0.01$ ). No significant changes were observed in the CF-treated mice following 2 weeks of treatment (Fig. 1D; P>0.05).

In addition, the serum insulin levels were determined. The diabetic mice demonstrated a significant decrease in 
Table I. Primer sequences for the analyzed genes.

\begin{tabular}{|c|c|c|c|}
\hline Gene & NCBI accession number & Primers $\left(5^{\prime} \rightarrow 3^{\prime}\right)$ & Size (bp) \\
\hline Ins-1 & NM_008386 & $\begin{array}{l}\text { F: CACTTCCTACCCCTGCTGG } \\
\text { R: ACCACAAAGATGCTGTTTGACA }\end{array}$ & 81 \\
\hline Ins-2 & NP_001172013.1 & $\begin{array}{l}\text { F: GCTTCTTCTACACACCCATGTC } \\
\text { R: AGCACTGATCTACAATGCCAC }\end{array}$ & 147 \\
\hline Neurog3 & NM_009719.6 & $\begin{array}{l}\text { F: GAAGCAGAAGTGGGTGAC } \\
\text { R: TGGGGAGTAGATAGAGCC }\end{array}$ & 384 \\
\hline Mafa & NM_194350.1 & $\begin{array}{l}\text { F: CAGCACCACCTGAACCCC } \\
\text { R: GGATGACCTCCTCCTTGC }\end{array}$ & 418 \\
\hline IL-6 & NM_031168 & $\begin{array}{l}\text { F: CTGCAAGAGACTTCCATCCAG } \\
\text { R: AGTGGTATAGACAGGTCTGTTGG }\end{array}$ & 131 \\
\hline TNF- $\alpha$ & NM_013693.2 & $\begin{array}{l}\text { F: GGGCTTCCAGAACTCCA } \\
\text { R: GCTACAGGCTTGTCACTCG }\end{array}$ & 213 \\
\hline CCL2 & NM_011333 & $\begin{array}{l}\text { F: TTAAAAACCTGGATCGGAACCAA } \\
\text { R: GCATTAGCTTCAGATTTACGGGT }\end{array}$ & 121 \\
\hline$\beta$-actin & NM_007393 & $\begin{array}{l}\text { F: GTGACGTTGACATCCGTAAAGA } \\
\text { R: GCCGGACTCATCGTACTCC }\end{array}$ & 245 \\
\hline
\end{tabular}

NCBI, National Center for Biotechnology Information; Ins-1, insulin 1; neurog3, neurogenin 3; mafa, v-maf musculoaponeurotic fibrosarcoma oncogene family, protein A; IL-6, interleukin 6; TNF- $\alpha$; tumor necrosis factor- $\alpha$; CCL2, chemokine (C-C motif) ligand 2; F, forward; R, reverse.

the serum insulin levels compared with the normal controls (Fig. 1E; $\mathrm{P}<0.01$ ). Compared with the diabetic control, the AC-, CF- and MF-treated mice demonstrated significant increases in serum insulin levels (Fig. 1E; $\mathrm{P}<0.01$ ). No significant difference was observed between the insulin levels of APS-treated mice those of the diabetic control mice (Fig. 1E; $\mathrm{P}>0.05)$.

OGTT. The OGTT effectively mimics the islet function and glucose metabolism. Following the oral administration of glucose, the diabetic mice demonstrated a significant increase in the AUC for blood glucose when compared with the normal controls (Fig. 2; $\mathrm{P}<0.01$ ). However, this increase was attenuated by $\mathrm{AC}, \mathrm{CF}$ and $\mathrm{MF}$ treatment (Fig. 2; $\mathrm{P}<0.05$ vs. the diabetic control). The APS-treated group demonstrated a significant increase in the AUC compared with the diabetic control following the oral administration of glucose in the diabetic mice (Fig. 2; $\mathrm{P}<0.01$ ). Taken together with the above results, the diabetic mice demonstrated the specific syndromes of type 1 diabetes, however AC treatment attenuated these syndromes and exerted synergic antidiabetic effects.

$H \& E$ staining. The primary function of the islet $\beta$-cells is to store and release insulin, to regulate glucose metabolism. Therefore, following treatment, the pathological changes of islet $\beta$-cells were assessed using H\&E staining. In the current study, STZ induced islet $\beta$-cells toxicity in the diabetic mice contributing to islet cell DNA damage compared with the normal control mice (Fig. 3A). Diabetic mice demonstrated a significant decrease in the size of islets of Langerhans. AC-, $\mathrm{CF}$ - and MF-treated mice demonstrated protective effects on the islet $\beta$-cells when compared with the diabetic control (Fig. 3A). However, no marked effect was demonstrated on the islet cells in APS-treated mice. Although Hawthorn demonstrated glucose-lowering effects in high-fat, diabetic mice in a previous study (10), no effects of restoring islet function were recorded. Therefore, the present results are the first, to the best of our knowledge, to demonstrate that $\mathrm{CF}$ induced the protective effects on islet cells.

Protein expression. In addition, the molecular mechanisms of the protective effects of $\mathrm{AC}$ on islet cells were determined at the protein level. PDX-1 in involved in pancreatic development, and $\beta$-cell function and survival (14). In the present study, the diabetic mice demonstrated significantly decreased PDX-1 protein expression levels in the pancreatic tissue when compared with the normal control (Fig. $3 \mathrm{~B}$ and $\mathrm{C} ; \mathrm{P}<0.01$ ). The AC-, CF- and MF-treated mice demonstrated significant increases in the PDX-1 protein expression levels compared with the diabetic control mice (Fig. $3 \mathrm{~B}$ and $\mathrm{C} ; \mathrm{P}<0.01$ ). However, no significant difference was observed between the protein expression levels of PDX-1 in the APS-treated mice and the diabetic control mice (Fig. 3B and C; $P>0.05$ ). These results indicated that the islet-restoring effect of $\mathrm{AC}$ may be mediated by upregulating PDX-1 expression.

In addition to the pancreatic islet cells, liver tissue cells are important in the regulation of glucose metabolism. pAMPK is an active form of AMPK that is responsible for enhancing glucose metabolism (15). In the present study, the expression level of pAMPK was significantly increased in the liver tissue of the AC-treated mice compared with the diabetic controls (Fig. 3D and E; P<0.01), a comparable increase was observed in the MF-treated mice $(\mathrm{P}<0.01)$. Furthermore, administration of $\mathrm{CF}$ demonstrated an increase in the pAMPK protein expression levels in the liver tissue of the diabetic mice $(\mathrm{P}<0.05)$. APS treatment, however, did 


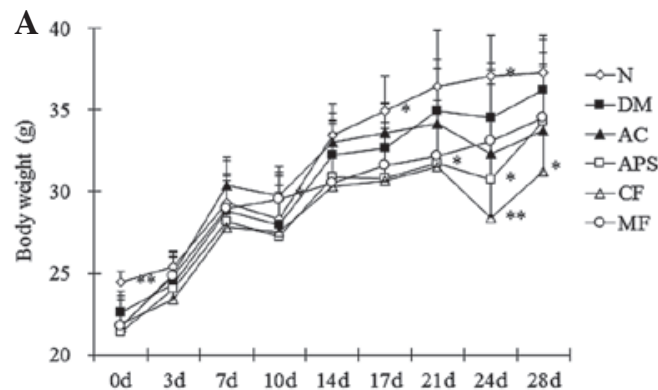

B
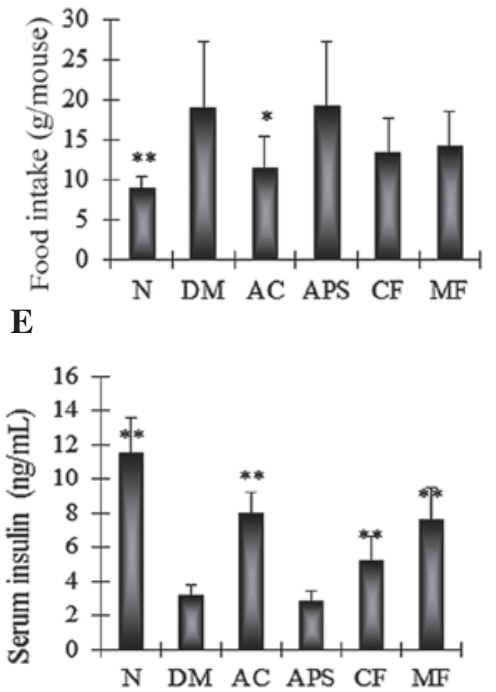

C
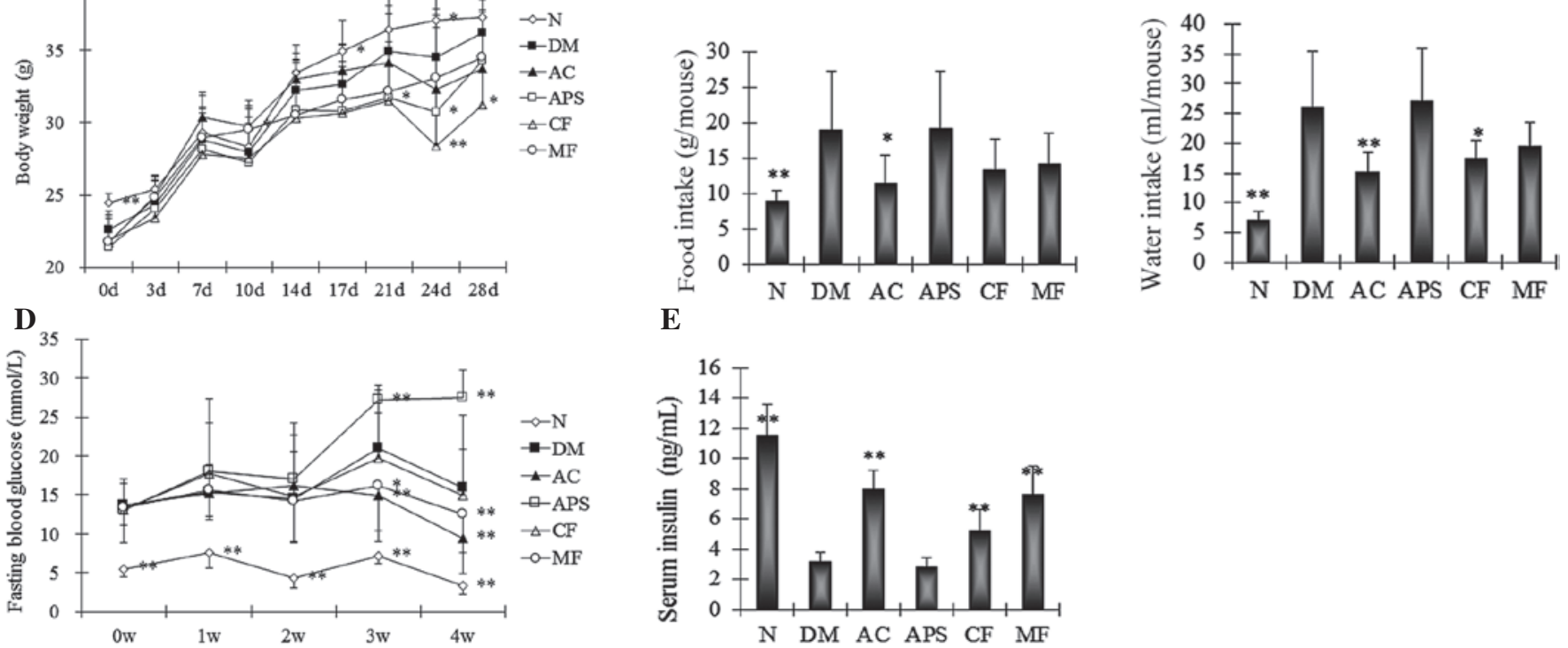

$-\mathrm{AC}$

APS

$\triangle \mathrm{MF}$

-DM

-APS

$\triangle \mathrm{CF}$

$-\mathrm{MF}$

Figure 1. Changes in the (A) body weight, (B) diet, (C) water intake, (D) fasting blood glucose and (E) serum insulin levels of the mice following 4 weeks of treatment. Data are expressed as the mean \pm standard deviation $(\mathrm{n}=10)$. ${ }^{*} \mathrm{P}<0.05,{ }^{* *} \mathrm{P}<0.01$ vs. the DM group. N, normal control; DM, diabetic control; AC, APS + CF-treated; APS, Astragalus polysaccharides; CF, Crataegus flavonoids; MF, metformin.

A

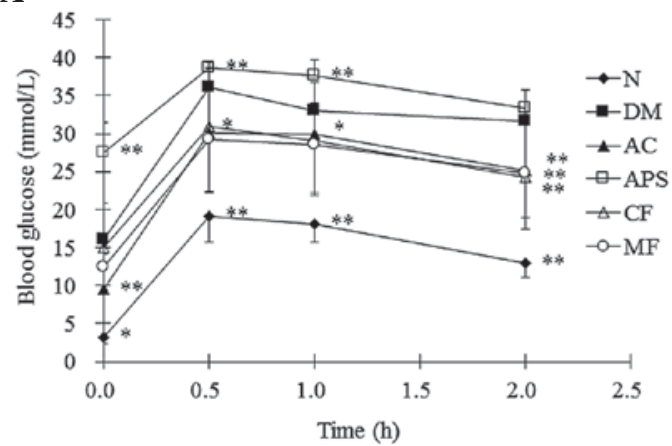

B

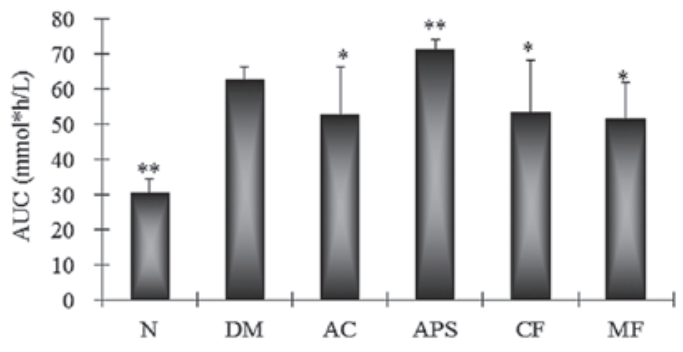

Figure 2. Temporal changes in the (A) blood glucose levels and (B) measurement of the AUC following oral administration of glucose. Data are expressed as the mean \pm standard deviation $(\mathrm{n}=6) .{ }^{*} \mathrm{P}<0.05,{ }^{* *} \mathrm{P}<0.01$ vs. the DM group. N, normal control; DM, diabetic control; AC, APS + CF-treated; APS, Astragalus polysaccharides; $\mathrm{CF}$, Crataegus flavonoids; MF, metformin; AUC, area under the curve.

not demonstrate a significant effect when compared with the diabetic control (Fig. 3D and E). The results indicate that the antidiabetic effects of AC may be mediated by the expression of $\mathrm{pAMPK}$.

mRNA expression levels. The gene expression of islet function- and inflammation-associated mRNAs in the pancreatic tissue of the mice was assessed using RT-qPCR. AC- and $\mathrm{CF}$-treated mice demonstrated a significant increase in the mRNA expression levels of Neurog3, Mafa, Ins-1 and Ins-2 in the pancreatic tissue compared with the diabetic control mice (Fig. 4A; P<0.01). APS treatment demonstrated an increase in the mRNA expression levels of Neurog3 and Mafa when compared with the diabetic control, however had less of an effect when compared with the $\mathrm{AC}$ - and $\mathrm{CF}$-treated mice (Fig. 4A). Furthermore, administration of MF demonstrated an increase in the Mafa, Ins-1 and Ins-2 mRNA expression levels compared with the diabetic control (Fig. 4A). Neurog3 and Mafa are responsible for islet development (16), and
Ins-1 and Ins-2 promote the synthesis of proinsulin. These results indicated that the restorative effects of $\mathrm{AC}$ on islet cells are mediated by upregulating the expression levels of islet function-associated genes.

Furthermore, the diabetic mice demonstrated significant increases in interleukin-6 (IL-6), tumor necrosis factor- $\alpha$ (TNF- $\alpha$ ) and chemokine (C-C motif) ligand 2 (CCL2) mRNA expression levels in the pancreatic tissues when compared with the normal control (Fig. 4B; $\mathrm{P}<0.01$ ). However, administration of $\mathrm{AC}$ and APS significantly reduced the expression levels of IL-6, TNF- $\alpha$ and CCL2 in the pancreatic tissues of the diabetic mice compared with those of the diabetic control mice (Fig. 4B; $\mathrm{P}<0.01$ ). Furthermore, $\mathrm{CF}$ treatment significantly reduced the mRNA expression levels of CCL2 compared with the diabetic control (Fig. 4B; $\mathrm{P}<0.01$ ). However, MF treatment significantly increased the mRNA expression levels of TNF- $\alpha$ and CCL2 compared with the diabetic control. These results indicated that the restorative effects of $\mathrm{AC}$ on islet cells involve the attenuation of inflammatory stress. 
A

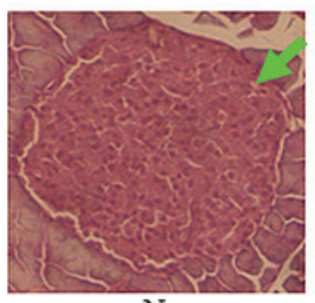

$\mathrm{N}$

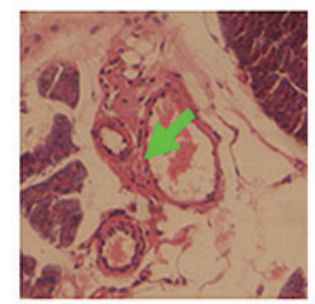

APS

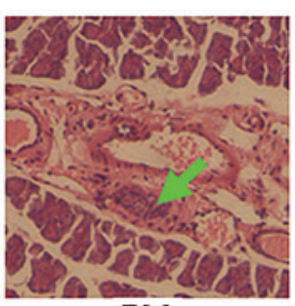

DM

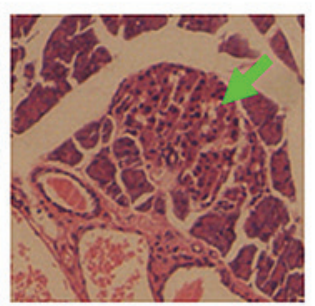

CF

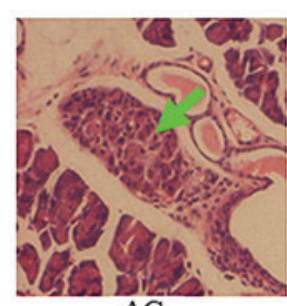

AC

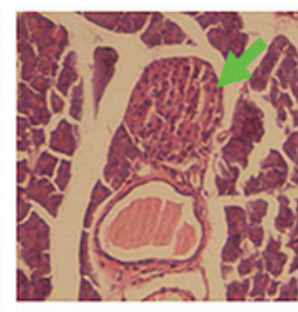

MF

B

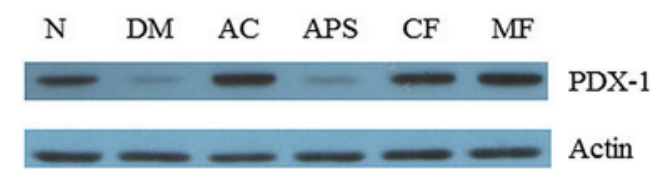

C

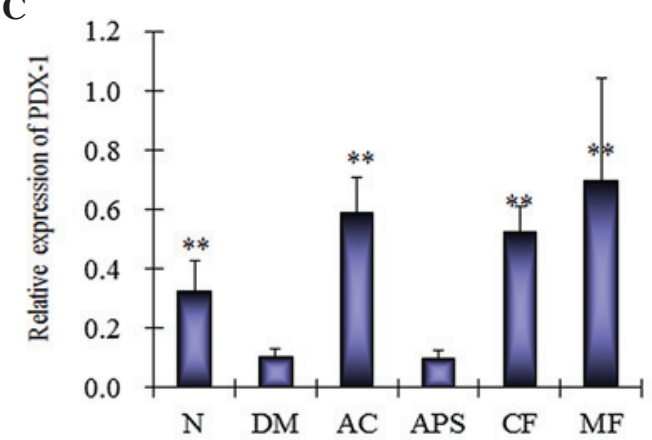

E

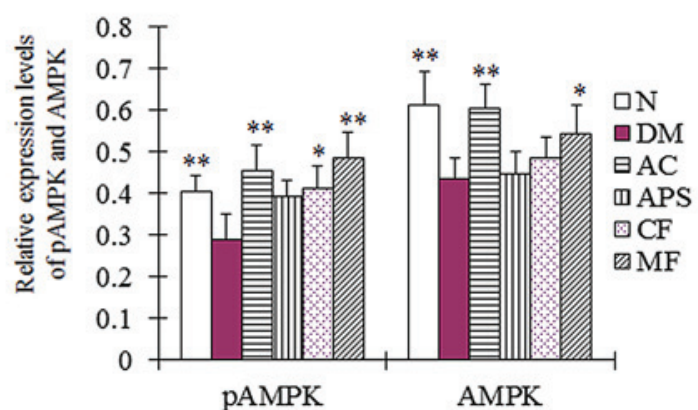

Figure 3. (A) Pathological changes (green arrows represent the islets of Langerhans) and (B and C) expression levels of PDX-1 in the islet cells of pancreatic tissue samples of mice following 4 weeks of treatment. (D and E) Expression levels of AMPK and pAMPK in mice liver tissue as determined by western blotting following 4 weeks of treatment. Data are expressed as the mean \pm standard deviation $(n=6) .{ }^{*} \mathrm{P}<0.05$, ${ }^{* *} \mathrm{P}<0.01$ vs. the $\mathrm{DM}$ group. $\mathrm{N}$, normal control; DM, diabetic control; AC, APS + CF-treated; APS, Astragalus polysaccharides; CF, Crataegus flavonoids; MF, metformin; PDX-1, pancreatic and duodenal homeobox-1; AMPK, adenosine 5'-monophosphate-activated protein kinase; p, phosphorylated.

\section{Discussion}

Astragalus and Hawthorn extracts in Chinese formulas are considered to have poor quality controls and variable clinical therapeutic effects. The present study selected refined active components to form a stable formula to guarantee a good quality control and stable therapeutic effects. In the current study, the combination treatment (AC) comprising APS and CF demonstrated synergic antidiabetic effects. Astragalus membranaceus and Hawthorn are the most frequently used traditional Chinese formulas to treat diabetes and hyperlipidemia, respectively $(7,9)$. However, in the current study Astragalus membranaceus or Hawthorn alone (using the typically prescribed active components) did not demonstrate the required antidiabetic effect. This indicates that active components from a single herb or other natural product may not be effective treatments; however, combining active components from various herbs or other natural sources may be a more promising strategy. Diabetes is a disease with complicated dysfunctions and requires combined therapeutic strategies. Therefore, it is hypothesized that an appropriate combination of different active components from natural products may aid in the prevention and treatment of diabetes.
In the current study, the antidiabetic effects and mechanisms of action of the combination therapy, APS + CF were investigated. The results demonstrated that AC had significant antidiabetic effects and it is proposed that the antidiabetic mechanisms of AC involve the restoration of pancreatic islet function and the improvement of glucose metabolism in the liver. PDX-1 serves an important role in pancreatic $\beta$-cell survival. The restoration effects of AC on $\beta$-islet cells may be mediated by the regulated expression of PDX-1. In addition, previous studies demonstrated that Hawthorn extracts and APS significantly increased AMPK phosphorylation $(10,17)$. However, in the present study, APS did not demonstrate a significant effect, which may have been due to variances in the different methods of extraction or dosages administered. In addition, AC significantly increased the expression level of AMPK in the samples of liver tissue from diabetic mice compared with the diabetic control mice. Thus, the AC combination treatment appeared to increase AMPK expression and promote phosphorylation of AMPK. Overall, this combination yielded synergic antidiabetic effects, however, the potential molecular mechanisms require further investigation.

Diabetes is an inflammatory disease (18). In the current study, AC and APS significantly decreased the mRNA expression levels of inflammatory factors, including IL- 6 , TNF- $\alpha$ 
A

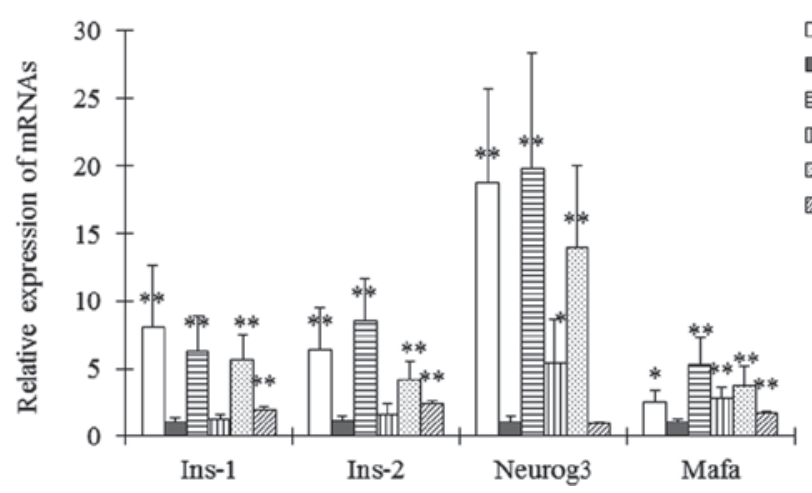

B

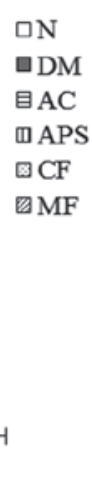

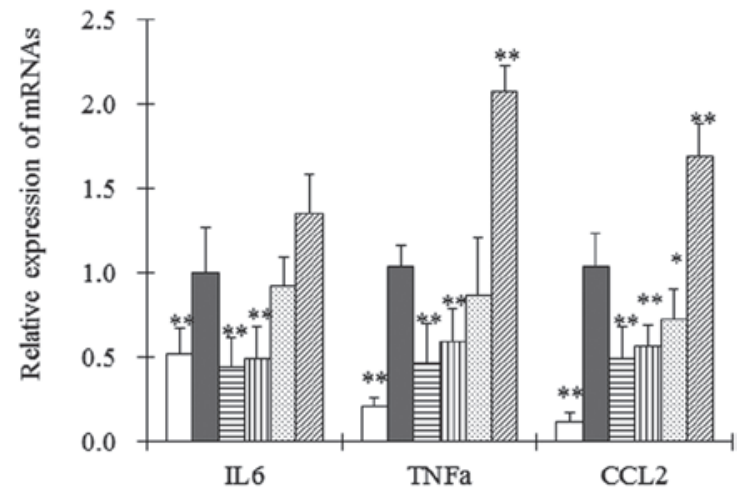

Figure 4. Relative mRNA expression levels of (A) Ins-1, Ins-2, Neurog3, Mafa and (B) IL-6, TNF- $\alpha$ and CCL2 in the pancreatic tissues of the mice assayed via reverse transcription-quantitative polymerase chain reaction following oral administration of the therapeutic agents for 4 weeks. Data are expressed as the mean \pm standard deviation $(\mathrm{n}=5) .{ }^{*} \mathrm{P}<0.05,{ }^{* *} \mathrm{P}<0.01$ vs. the DM group. N, normal control; DM, diabetic control; AC, APS + CF-treated; APS, Astragalus polysaccharides; CF, Crataegus flavonoids; MF, metformin; Ins-1, insulin 1; neurog3, neurogenin 3; mafa, v-maf musculoaponeurotic fibrosarcoma oncogene family, protein A; IL-6, interleukin 6; TNF- $\alpha$; tumor necrosis factor- $\alpha$; CCL2, chemokine (C-C motif) ligand 2.

and CCL2 in the pancreatic tissues. CF treatment markedly decreased the expression level of CCL2, however, MF treatment increased the expression levels of IL-6, TNF- $\alpha$ and CCL2. A previous study demonstrated the direct inhibition of APS on the lipopolysaccharide-induced inflammatory responses in monocytes or macrophages (19). In the present study, however, APS did not protect the $\beta$-cells as previously reported (20). This result may be due to the fact that a lower dose of APS could not control the fasting blood glucose, thus, the islet $\beta$-cells were subjected to long-term, severe glucotoxicity. However, the present study proposes that the antidiabetic effects of AC are mediated by its anti-inflammatory effects.

The administration of CF demonstrated no significant effects on the inhibition of the expression of inflammatory factors IL- 6 and TNF- $\alpha$ in the present study, although the flavonoids from Hawthorn leaves exerted anti-inflammatory effects in a previous study (21). Flavonoids extracted from different sources or analyzed at different dosages may have variable components and effects. In the current study, CF appeared to upregulate the expression levels of PDX-1, Mafa, Neurog3, Ins-1 and Ins-2. A previous study demonstrated that the combination of PDX-1, Mafa and Neurog3 markedly induced insulin biosynthesis in various non- $\beta$-cells (22). The upregulation of Ins- 1 or Ins- 2 may be triggered by the increased expression levels of Mafa, PDX-1 and Neurog3. Therefore, CF may restore the islet cell function by regulating the expression of the above-mentioned factors. Thus, the anti-inflammatory activities of APS combined with the islet-restoring activities of CF contributed to improved antidiabetic effects of AC treatment, when compared with APS or $\mathrm{CF}$ treatment alone.

In conclusion, to the best of our knowledge, this is the first study to suggest that combining APS and CF may be a promising treatment for diabetes, as demonstrated by the synergic antidiabetic effects. This combination may facilitate the rehabilitation of islet cell function by upregulating PDX-1 expression and promoting the metabolism of liver glucose via the promotion of AMPK phosphorylation (Fig. 5). These effects of AC may be useful for the treatment of type 1 or 2 diabetes, as the patients are frequently subjected to impaired

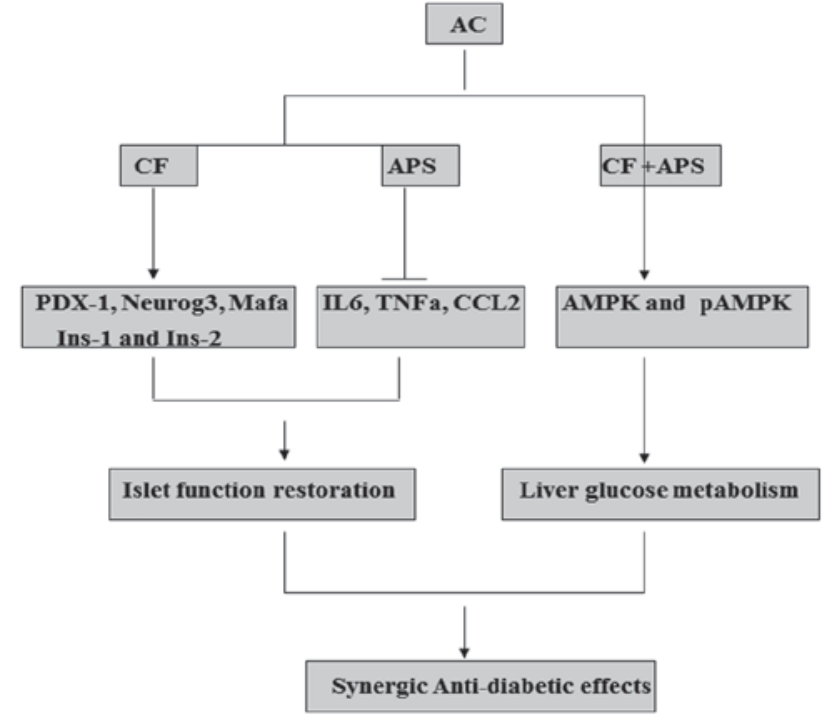

Figure 5. Synergic antidiabetic effects and potential mechanisms of action of APS and CF in the treatment of diabetes. AC, APS + CF-treated; APS, Astragalus polysaccharides; CF, Crataegus flavonoids; PDX-1, pancreatic and duodenal homeobox-1; neurog3, neurogenin 3; mafa, v-maf musculoaponeurotic fibrosarcoma oncogene family, protein A; Ins-1/2, insulin 1/2; IL-6, interleukin 6; TNF- $\alpha$; tumor necrosis factor- $\alpha$; CCL2, chemokine (C-C motif) ligand 2; AMPK, adenosine 5'-monophosphate-activated protein kinase; $p$, phosphorylated.

islet cell function and disordered extrapancreatic metabolism. In addition, the anti-inflammatory activity of APS and the islet-restoring effects of $\mathrm{CF}$ contributed to the synergic effects of $\mathrm{AC}$ on protecting islet function. Further investigations are required to establish the potential molecular mechanisms or compounds that contribute to this effect.

\section{Acknowledgements}

The present study was supported by the National Natural Science Foundation of China (grant no. 81373460), the Natural Science Foundation of Guangdong Province (grant 
no. 2014A030313744), the Shenzhen Science and Technology R\&D Foundation (grant no. SGLH20121008144756945) and the China Scholarship Council (grant no. 201308440130).

\section{References}

1. Yang W, Lu J, Weng J, Jia W, Ji L, Xiao J, Shan Z, Liu J, Tian H, Ji Q, et al: China National Diabetes and Metabolic Disorders Study Group: Prevalence of diabetes among men and women in China. N Engl J Med 362: 1090-1101, 2010.

2. Konig M, Lamos EM, Stein SA and Davis SN: An insight into the recent diabetes trials: What is the best approach to prevent macrovascular and microvascular complications? Curr Diabetes Rev 9: 371-381, 2013.

3. Gajos G, Piłaciński S and Zozulińska-Ziółkiewicz D: Controversies in diabetes in 2013 - a brief update. Adv Clin Exp Med 22: 777-784, 2013.

4. Vaz JA and Patnaik A: Diabetes mellitus: Exploring the challenges in the drug development process. Perspect Clin Res 3: 109-112, 2012.

5. American Diabetes Association: Approaches to glycemic treatment. Diabetes Care 38(Suppl 1): S41-48, 2015.

6. Sun Y, Fan L, Meng J, Zhang F, Zhang D and Mei Q: Should GLP-1 receptor agonists be used with caution in high risk population for colorectal cancer? Med Hypotheses 82: 255-256, 2014

7. Xie W, Zhao Y and Zhang Y: Traditional Chinese medicines in treatment of patients with type 2 diabetes mellitus. Evid Based Complement Alternat Med 2011: 726723, 2011.

8. Agyemang K, Han L, Liu E, Zhang Y, Wang T and Gao X: Recent advances in Astragalus membranaceus anti-diabetic research: Pharmacological effects of its phytochemical constituents. Evid Based Complement Alternat Med 2013: 654643, 2013.

9. Xie W, Zhao Y and Du L: Emerging approaches of traditional Chinese medicine formulas for the treatment of hyperlipidemia. J Ethnopharmacol 140: 345-367, 2012.

10. Shih CC, Lin CH, Lin YJ and Wu JB: Validation of the antidiabetic and hypolipidemic effects of Hawthorn by assessment of gluconeogenesis and lipogenesis related genes and AMP-activated protein kinase phosphorylation. Evid Based Complement Alternat Med 2013: 597067, 2013.

11. Wang J, Wang YY and Yang G: Methods and modes about the theory of traditional Chinese prescription composition. Zhongguo Zhong Yao Za Zhi 30: 6-8, 11, 2005 (In Chinese).
12. Xie W, Zhao Y, Gu D, Du L, Cai G, Zhang Y: Scorpion in Combination with Gypsum: Novel Antidiabetic Activities in Streptozotocin-Induced Diabetic Mice by Up-Regulating Pancreatic PPAR $\gamma$ and PDX-1 Expressions. Evid Based Complement Alternat Med 2011:683561, 2011.

13. Livak KJ and Schmittgen TD: Analysis of relative gene expression data using real-time quantitative PCR and the 2(-Delta Delta C(T)) Method. Methods 25: 402-408, 2001

14. Hayes HL, Moss LG, Schisler JC, Haldeman JM, Zhang Z, Rosenberg PB, Newgard CB and Hohmeier HE: Pdx-1 activates islet $\alpha$ - and $\beta$-cell proliferation via a mechanism regulated by transient receptor potential cation channels 3 and 6 and extracellular signal-regulated kinases 1 and 2. Mol Cell Biol 33: 4017-4029, 2013.

15. Winder WW: AMP-activated protein kinase: Possible target for treatment of type 2 diabetes. Diabetes Technol Ther 2: 441-448, 2000.

16. Zaldumbide A, Carlotti F, Gonçalves MA, Knaän-Shanzer S, Cramer SJ, Roep BO, Wiertz EJ and Hoeben RC: Adenoviral vectors stimulate glucagon transcription in human mesenchymal stem cells expressing pancreatic transcription factors. PLoS One 7: e48093, 2012.

17. Zou F, Mao XQ, Wang N, Liu J and Ou-Yang JP: Astragalus polysaccharides alleviates glucose toxicity and restores glucose homeostasis in diabetic states via activation of AMPK. Acta Pharmacol Sin 30: 1607-1615, 2009.

18. Xie W and Du L: Diabetes is an inflammatory disease: Evidence from traditional Chinese medicines. Diabetes Obes Metab 13: 289-301, 2011.

19. Lu J, Chen X, Zhang Y, Xu J, Zhang L, Li Z, Liu W, Ouyang J, Han S and He X: Astragalus polysaccharide induces anti-inflammatory effects dependent on AMPK activity in palmitate-treated RAW264.7 cells. Int J Mol Med 31: 1463-1470, 2013.

20. Li RJ, Qiu SD, Chen HX, Tian H and Liu GQ: Effect of Astragalus polysaccharide on pancreatic cell mass in type 1 diabetic mice. Zhongguo Zhong Yao Za Zhi 32: 2169-2173, 2007 (In Chinese).

21. Fu JH, Zheng YQ, Li P, Li XZ, Shang XH and Liu JX: Hawthorn leaves flavonoids decreases inflammation related to acute myocardial ischemia/reperfusion in anesthetized dogs. Chin J Integr Med 19: 582-588, 2013.

22. Kaneto H, Matsuoka TA, Katakami $N$ and Matsuhisa M: Combination of MafA, PDX-1 and NeuroD is a useful tool to efficiently induce insulin-producing surrogate beta-cells. Curr Med Chem 16: 3144-3151, 2009. 